\title{
Utilization of the EG8010 Microcontroller to Construct a Pure Sine Inverter Using the Single Phase Sinusoidal Inverter ASIC (Application Specific Integrated Circuit) Method
}

\author{
Kurnia Brahmana $^{1 *}$ and Syukur Fransiskus Jaya Hulu ${ }^{2}$ \\ ${ }^{1,2}$ Department of Physics, Faculty of Mathematics and Natural Science, Universitas Sumatera Utara \\ 20155, Indonesia
}

\begin{abstract}
A pure sine inverter has been successfully designed by utilizing the EG8010 microcontroller which is used as an alternative energy source when the main power grid is cut. This pure sine inverter tool functions to stop the conversion when the power drops from the minimum value. This tool consists of a microcontroller to run the DC to AC converter instructions for 380 volts. Meanwhile, DC functions as a voltage source that provides the overall voltage. The signal waves that have been converted from DC to AC on the EG8010 Module are needed to control FET which functions as a signal amplifier. This signal is used to drive four FET n-channel and IRF480 which work alternately in sending data to the power FET to form an H-Bridge bridge and the output signal from the H-Bridge. Furthermore, the H-Bridge bridge and the output signal from the H-Bridge are sent through the transformer, so that the final output signal is a pure DC to AC sine wave. The resulting pure sine wave dc to ac can provide a maximum power of 240 watts.
\end{abstract}

Keyword: Microcontroller EG8010, IRF480, Power H-Bridge, Mosfet, FET

Received 1 July 2020 | Revised [15 July 2020] | Accepted [27 August 2020]

\section{Introduction}

Utilization of renewable energy is one of the fastest growing solutions in people's lives, especially in the industrial sector. This occurs due to the increasing demand for energy, so that people are looking for solutions to use renewable energy for low cost and environmentally friendly. Solar energy is one of the potential renewable energy sources because of its availability, convenience and use, which is cheaper and optimal operation. Solar energy can be converted into electrical energy so that this condition can be a good solution to overcome the electricity crisis in a country. An AC/ DC power inverter is required to convert the DC voltage collected by the photovoltaic cell into AC voltage. Square-wave inverters have easier and cheaper circuits, but square-wave inverters will lag behind in the process of performance. Then

*Corresponding author at: Jl. Bioteknologi No.1 Kampus USU, Medan, Indonesia, 20155

E-mail address: kurniabrahmana@usu.ac.id 
the output data is compared with a sine wave inverter. Sine wave inverter can provide pure sine wave output. Pure sine wave operates in near perfect efficiency, and maximum output [1-6].

Pure sine wave inverter is a tool to convert DC voltage into pure AC in operating AC loads. An inverter cannot generate power but an inverter can only convert DC power into AC power. Pure sine wave inverter is a type of inverter that does not have harmful harmonics for all types of AC loads $[1,3]$. There are several methods to generate sinusoidal waves, namely, the Synchronous Pulse Width Modulation (SPWM) method, and the Single Phase Sinusoidal Method (ASIC). The ASIC method has several advantages, one of which is an easier processing, the components used in this method are widely sold, and the output is efficient [7].

Sine wave is one of the basic waveforms that is often used to operate electrical equipment. However, the majority of the current sine waves are supplied only by state electricity companies or can be generated using a generator. So there is a problem that the energy crisis and the cost of supplying electrical energy are getting higher. In this condition, especially in areas that have not been reached by electricity, it is necessary to build a sine wave generator at a lower cost, because the price of inverters sold in companies is relatively expensive [6]. One solution is to use a battery with several electronic circuits added so that it can produce pure sine waves that can be applied to everyday electrical equipment. In this article, an innovation will be made in the manufacture of a pure sine inverter with the single phase sinusoidal inverter ASIC method (Application Specific Integrated Circuit) by utilizing the EG8010 Microcontroller [8-14]. This research is expected to make a pure sine inverter tool that is effective and efficient so that it can be applied optimally in everyday life.

\section{Methods}

The process of designing a pure sine wave inverter from the EG8010 Microcontroller can be seen in Figure 1 and Figure 2.

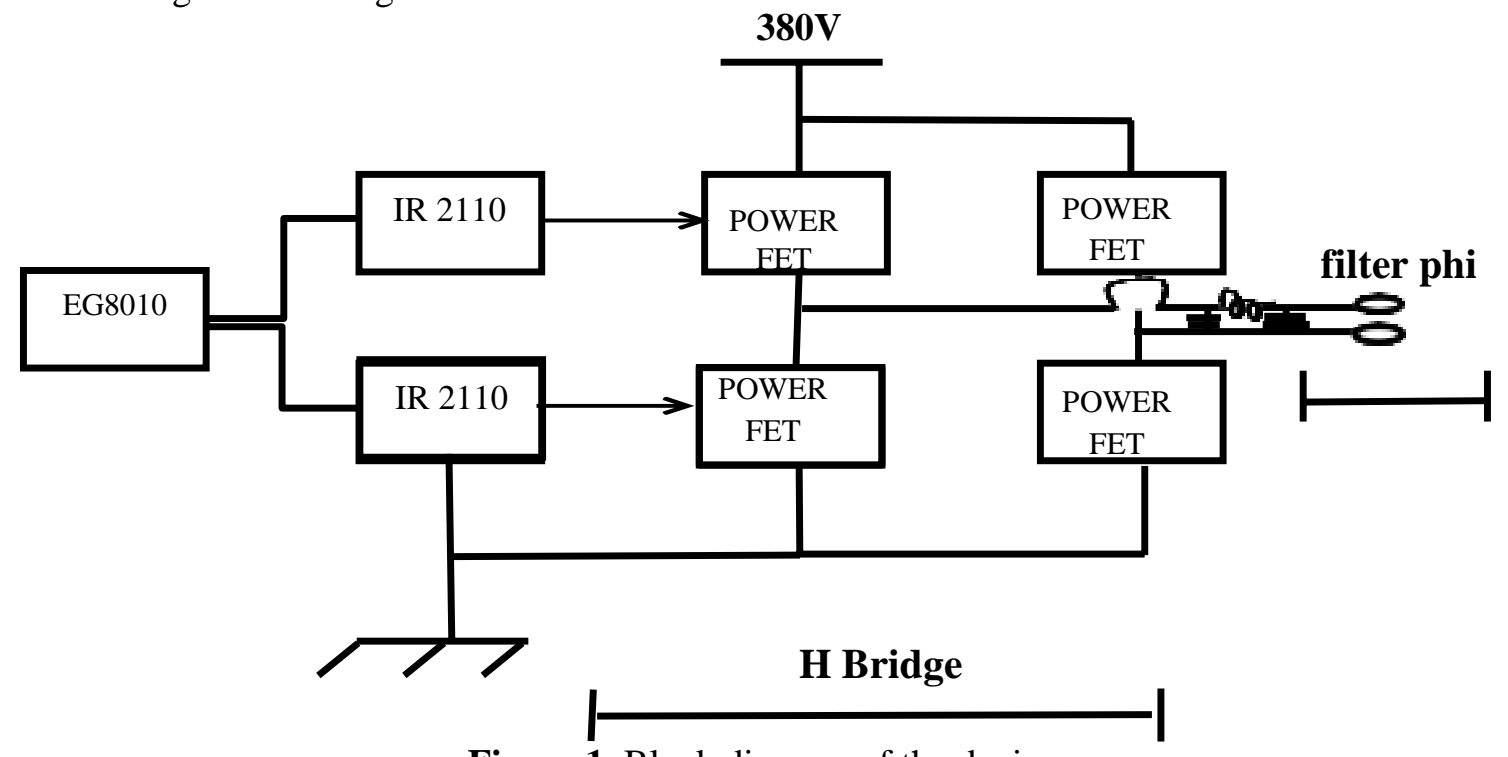

Figure 1. Block diagram of the device 
Function of each block of block diagram

1. EG8010 block: EG8010 is a digital pure sine wave inverter ASIC (Application Specific Integrated Circuit) with complete built-in dead time control functions.

2. IR 2110 Block: Controls the voltage and current between the POWER FET

3. Power FET block: Sinusoidal signal amplifier.

4. Phi filter: Filters all excess noise above the critical frequency as close as possible to the desired frequency.

5. 380V DC: The source of the output voltage will be $220 \mathrm{VAC}$ and the power to run the circuit on the Microcontroller.

6. 220V AC: The output of the inverter circuit.

7. H Bridge: An H-Bridge or full-bridge converter that has a toggle configuration, consisting of four switches in settings and used as a switch controller.
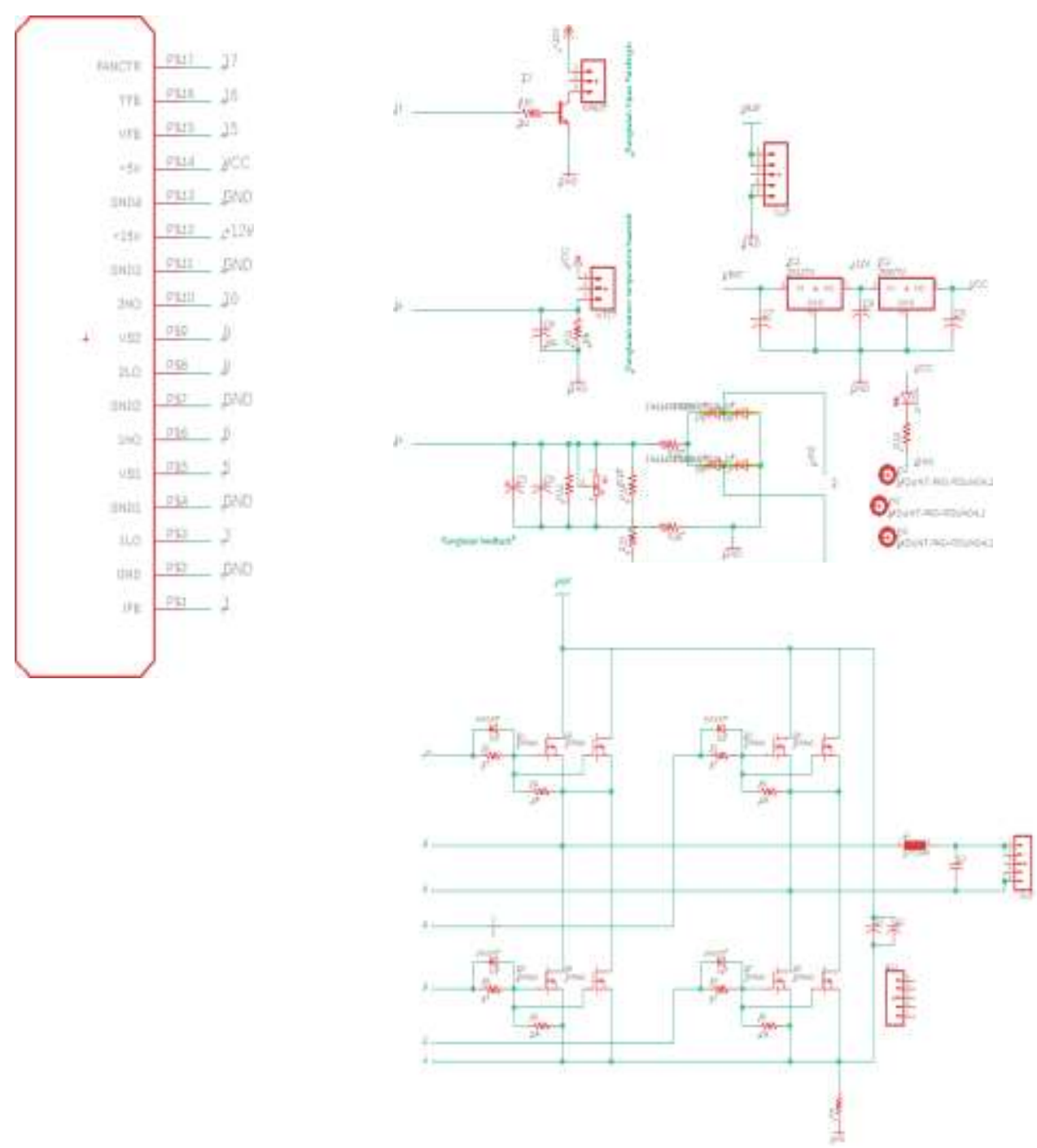

Figure 2. Whole Circuit Trial 


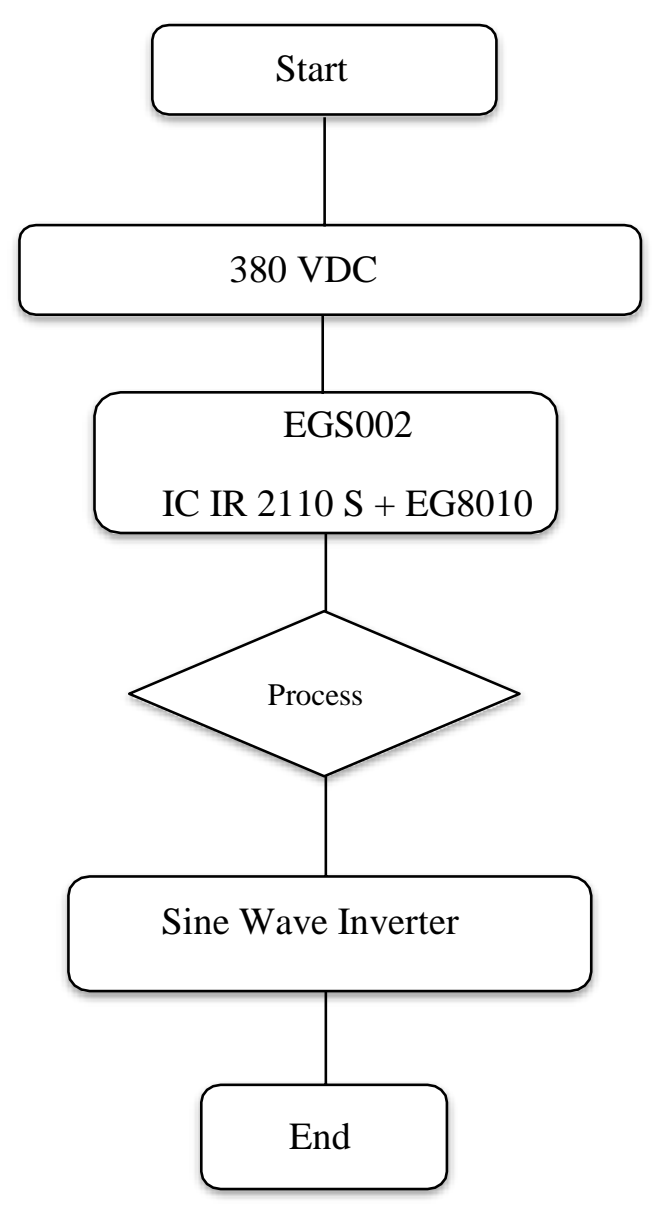

Figure 3. Flowchart

\section{Result and Discussion}

The AC voltage sensor emits an analog signal that the reading by the sensor is directly received by Arduino via the ADC (Analog Digital Converter) pin, which in this study uses pin A1 on the Arduino board. In this study, resistors were used $670 \mathrm{k} \Omega$ and $10 \mathrm{k} \Omega$ to a maximum voltage that can be read at 340 volts.

The ACS712 analog value will change according to the current applied and the bias of the circuit. If the ACS712 is connected in a forward biased state according to pins + IP and -IP, the resulting analog value will increase, whereas if connected in a reverse bias state the resulting analog value will decrease. In this research, the current sensor used is the current sensor version ACS712-5A with a range that can be read from -5 A to $5 \mathrm{~A}$. The ACS712-5A current sensor has a sensitivity of $185 \mathrm{mV} / \mathrm{A}$ and a better resolution than other versions. 
Table 1. Testing Data for Pure Sine Inverter DC to AC at 40 Watt Load

\begin{tabular}{cccccccc}
\hline No & $\mathrm{V}_{\text {in }}(\mathrm{V})$ & $\mathrm{I}_{\text {in }}(\mathrm{A})$ & $\mathrm{V}_{\text {out }}(\mathrm{V})$ & $\mathrm{I}_{\text {out }}(\mathrm{A})$ & $\mathrm{P}_{\text {In }}$ & $\mathrm{P}_{\text {Out }}$ & $\begin{array}{c}\text { Efficiency } \\
(\%)\end{array}$ \\
\hline 1 & 13.65 & 2.92 & 215.2 & 0.15 & 39.858 & 32.280 & 80.9875 \\
2 & 13.64 & 2.86 & 215.9 & 0.14 & 39.010 & 30.226 & 77.4819 \\
3 & 13.65 & 2.94 & 215.7 & 0.15 & 40.131 & 32.355 & 80.6234 \\
4 & 13.63 & 2.83 & 215.9 & 0.14 & 38.573 & 30.226 & 78.3607 \\
5 & 13.65 & 2.94 & 215.2 & 0.15 & 40.131 & 32.280 & 80.4365 \\
6 & 13.65 & 2.96 & 215.2 & 0.15 & 40.404 & 32.280 & 79.8930 \\
7 & 13.63 & 2.93 & 215.9 & 0.14 & 39.936 & 30.226 & 75.6862 \\
8 & 13.65 & 2.83 & 215.8 & 0.15 & 38.629 & 32.370 & 83.7960 \\
9 & 13.65 & 2.92 & 215.7 & 0.14 & 39.858 & 30.198 & 75.7639 \\
10 & 13.63 & 2.91 & 215.1 & 0.14 & 39.663 & 30.114 & 75.9240 \\
11 & 13.65 & 2.94 & 215.5 & 0.15 & 40.131 & 32.325 & 80.5487 \\
12 & 13.65 & 2.94 & 215.5 & 0.15 & 40.131 & 32.325 & 80.5487 \\
13 & 13.66 & 2.91 & 215.7 & 0.14 & 39.751 & 30.198 & 75.9686 \\
14 & 13.65 & 2.94 & 215.6 & 0.15 & 40.131 & 32.340 & 80.5860 \\
15 & 13.65 & 2.93 & 215.8 & 0.14 & 39.995 & 30.212 & 75.5403 \\
16 & 13.67 & 2.87 & 215.9 & 0.15 & 39.233 & 32.385 & 82.5455 \\
\hline \multicolumn{7}{c}{ Average } \\
\hline
\end{tabular}

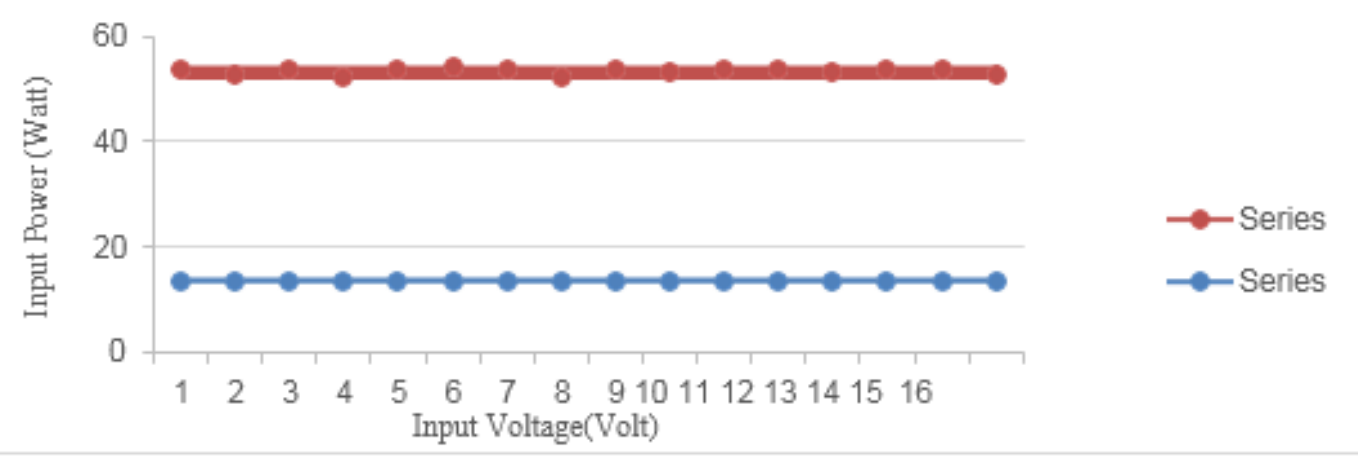

Figure 4. Graph of V Input vs Input Power with a load of 40 Watts

Table 2. Testing Data for Pure Sine Inverter DC to AC at 75 Watt Load

\begin{tabular}{cccccccc}
\hline No & $\begin{array}{c}\mathrm{V}_{\text {in }} \\
(\mathrm{V})\end{array}$ & $\mathrm{I}_{\text {in }}(\mathrm{A})$ & $\mathrm{V}_{\text {out }}(\mathrm{V})$ & $\mathrm{I}_{\text {out }}(\mathrm{A})$ & $\mathrm{P}_{\text {In }}$ & $\mathrm{P}_{\text {Out }}$ & $\begin{array}{c}\text { Efficiency } \\
(\%)\end{array}$ \\
\hline 1 & 13.45 & 5.55 & 200.82 & 0.28 & 74.648 & 56.229 & 75.3268 \\
2 & 13.48 & 5.52 & 211.38 & 0.27 & 74.410 & 57.073 & 76.7006 \\
3 & 13.44 & 5.50 & 211.38 & 0.27 & 73.920 & 57.073 & 77.2086 \\
4 & 13.48 & 5.51 & 211.50 & 0.28 & 74.275 & 59.220 & 79.7310 \\
5 & 13.40 & 5.49 & 200.51 & 0.28 & 73.566 & 56.143 & 76.3162 \\
6 & 13.42 & 5.47 & 211.50 & 0.27 & 73.407 & 57.105 & 77.7919 \\
7 & 13.46 & 5.48 & 211.48 & 0.28 & 73.761 & 59.214 & 80.2790 \\
8 & 13.44 & 5.54 & 211.60 & 0.28 & 74.458 & 59.248 & 79.5728 \\
9 & 13.49 & 5.48 & 211.70 & 0.26 & 73.925 & 55.042 & 74.4563 \\
10 & 13.42 & 5.46 & 200.50 & 0.29 & 73.273 & 58.145 & 79.3537 \\
11 & 13.44 & 5.48 & 211.50 & 0.28 & 73.651 & 59.220 & 80.4060 \\
12 & 13.40 & 5.38 & 200.20 & 0.29 & 72.092 & 58.058 & 80.5332 \\
13 & 13.48 & 5.50 & 211.70 & 0.26 & 74.140 & 55.042 & 74.2406 \\
14 & 13.41 & 5.40 & 211.20 & 0.27 & 72.414 & 57.024 & 78.7472 \\
15 & 13.40 & 5.56 & 211.50 & 0.28 & 74.504 & 59.220 & 79.4856 \\
16 & 13.46 & 5.54 & 211.65 & 0.28 & 74.568 & 59.262 & 79.4733 \\
\hline \multicolumn{7}{c}{ Average } \\
\hline \multicolumn{7}{c}{}
\end{tabular}




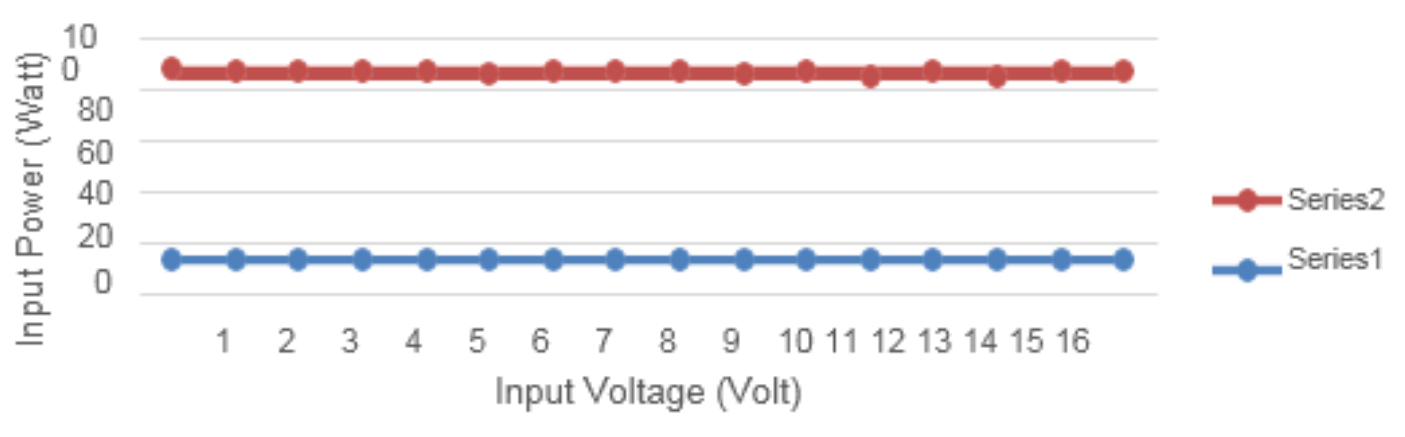

Figure 5. Input Voltage vs Input Power Graph, 75 Watt load

Table 3. Testing Data for Pure Sine Inverter DC to AC at 100 Watt Load

\begin{tabular}{cccccccc}
\hline No & $\mathrm{V}_{\text {in }}(\mathrm{V})$ & $\mathrm{I}_{\text {in }}(\mathrm{A})$ & $\mathrm{V}_{\text {out }}(\mathrm{V})$ & $\mathrm{I}_{\text {out }}(\mathrm{A})$ & $\mathrm{P}_{\text {In }}$ & $\mathrm{P}_{\text {Out }}$ & $\begin{array}{c}\text { Efficiency } \\
(\%)\end{array}$ \\
\hline 1 & 13.25 & 7.42 & 205.2 & 0.38 & 98.315 & 77.976 & 79.31241 \\
2 & 13.26 & 7.46 & 205.1 & 0.37 & 98.920 & 75.887 & 76.71584 \\
3 & 13.18 & 7.40 & 205.3 & 0.37 & 97.532 & 75.961 & 77.88316 \\
4 & 13.20 & 7.50 & 205.5 & 0.38 & 99.000 & 78.090 & 78.87879 \\
5 & 13.22 & 7.30 & 205.3 & 0.36 & 96.506 & 73.908 & 76.58384 \\
6 & 13.24 & 7.40 & 205.4 & 0.35 & 97.976 & 71.890 & 73.37511 \\
7 & 13.20 & 7.20 & 205.2 & 0.34 & 95.040 & 69.768 & 73.40909 \\
8 & 13.18 & 7.40 & 205.3 & 0.33 & 97.532 & 67.749 & 69.46336 \\
9 & 13.26 & 7.40 & 205.5 & 0.38 & 98.124 & 78.090 & 79.58298 \\
10 & 13.16 & 7.44 & 205.6 & 0.39 & 97.910 & 80.184 & 81.89528 \\
11 & 13.18 & 7.46 & 205.6 & 0.38 & 98.323 & 78.128 & 79.46072 \\
12 & 13.22 & 7.39 & 200.7 & 0.38 & 97.696 & 76.266 & 78.06477 \\
13 & 13.26 & 7.45 & 205.3 & 0.37 & 98.787 & 75.961 & 76.89372 \\
14 & 13.28 & 7.43 & 205.6 & 0.38 & 98.670 & 78.128 & 79.18079 \\
15 & 13.20 & 7.20 & 200.2 & 0.40 & 95.040 & 80.080 & 84.25926 \\
16 & 13.21 & 7.42 & 205.1 & 0.39 & 98.018 & 79.989 & 81.60627 \\
& \multicolumn{7}{c}{ Average } \\
\hline \multicolumn{7}{c}{} \\
\hline
\end{tabular}

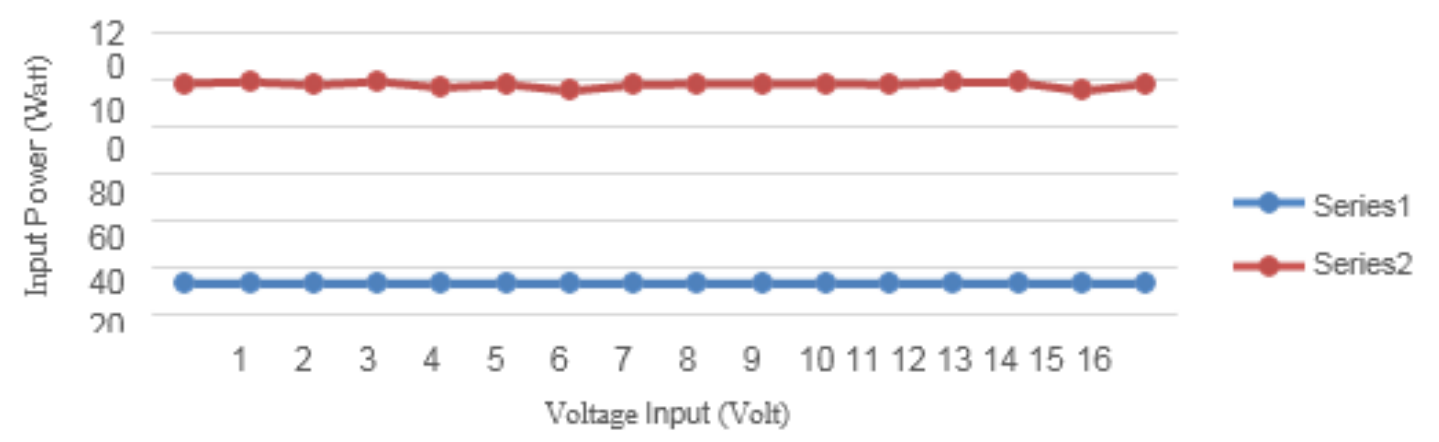

Figure 6. Input Voltage vs Input Power Graph, 100 Watt load 
Table 4. Testing Data for Pure Sine Inverter DC to AC at 150 Watt Load

\begin{tabular}{cccccccc}
\hline $\mathrm{No}$ & $\mathrm{V}_{\text {in }}(\mathrm{V})$ & $\mathrm{I}_{\text {in }}(\mathrm{A})$ & $\mathrm{V}_{\text {out }}(\mathrm{V})$ & $\mathrm{I}_{\text {out }}(\mathrm{A})$ & $\mathrm{P}_{\text {In }}$ & $\mathrm{P}_{\text {Out }}$ & Efficiency (\%) \\
\hline 1 & 12.88 & 11.20 & 190.3 & 0.55 & 144.256 & 104.665 & 72.55504 \\
2 & 12.90 & 11.22 & 192.5 & 0.58 & 144.738 & 111.650 & 77.13938 \\
3 & 12.86 & 11.26 & 192.3 & 0.59 & 144.804 & 113.457 & 78.35233 \\
4 & 12.84 & 11.20 & 192.7 & 0.57 & 143.808 & 109.839 & 76.37892 \\
5 & 12.84 & 11.40 & 192.3 & 0.58 & 146.376 & 111.534 & 76.19692 \\
6 & 12.88 & 11.25 & 192.4 & 0.59 & 144.900 & 113.516 & 78.34092 \\
7 & 12.90 & 11.26 & 192.6 & 0.60 & 145.254 & 115.560 & 79.55719 \\
8 & 12.84 & 11.20 & 188.3 & 0.60 & 143.808 & 112.980 & 78.56308 \\
9 & 12.83 & 11.20 & 192.6 & 0.58 & 143.696 & 111.708 & 77.73912 \\
10 & 12.88 & 11.28 & 190.8 & 0.60 & 145.286 & 114.480 & 78.79609 \\
11 & 12.90 & 11.20 & 192.6 & 0.58 & 144.480 & 111.708 & 77.31728 \\
12 & 12.92 & 11.25 & 190.7 & 0.60 & 145.350 & 114.420 & 78.72033 \\
13 & 12.90 & 11.18 & 192.4 & 0.58 & 144.222 & 111.592 & 77.37516 \\
14 & 12.80 & 11.16 & 192.6 & 0.56 & 142.848 & 107.856 & 75.50403 \\
15 & 12.84 & 11.22 & 192.3 & 0.54 & 144.065 & 103.842 & 72.08006 \\
16 & 12.81 & 11.16 & 192.5 & 0.58 & 142.960 & 111.650 & 78.09899 \\
\hline \multicolumn{7}{c}{ Average }
\end{tabular}

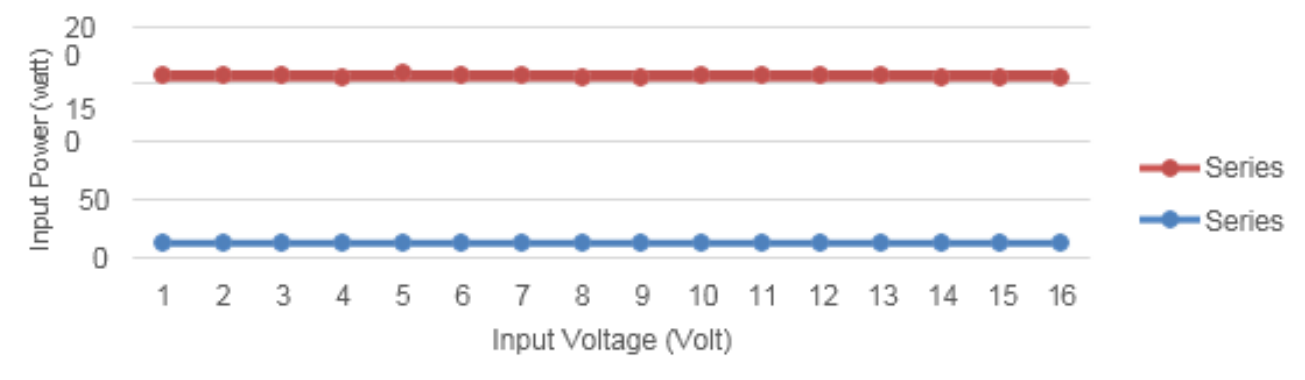

Figure 7. Graph V Input vs Input Power with 150 Watt load

Table 5. Testing Data for Pure Sine Inverter DC to AC at 200 Watt Load

\begin{tabular}{cccccccc}
\hline No & $\mathrm{V}_{\text {in }}(\mathrm{V})$ & $\mathrm{I}_{\text {in }}(\mathrm{A})$ & $\mathrm{V}_{\text {out }}(\mathrm{V})$ & $\mathrm{I}_{\text {out }}(\mathrm{A})$ & $\mathrm{P}_{\text {In }}$ & $\mathrm{P}_{\text {Out }}$ & Efficiency (\%) \\
\hline 1 & 12.60 & 15.72 & 186.40 & 0.80 & 198.0720 & 149.1200 & 75.28575 \\
2 & 12.64 & 15.70 & 186.10 & 0.82 & 198.4480 & 152.6020 & 76.89773 \\
3 & 12.64 & 15.72 & 186.30 & 0.83 & 198.7008 & 154.6290 & 77.82002 \\
4 & 12.68 & 15.72 & 186.38 & 0.80 & 199.3296 & 149.1040 & 74.80274 \\
5 & 12.63 & 15.70 & 186.60 & 0.80 & 198.2910 & 149.2800 & 75.28330 \\
6 & 12.65 & 15.78 & 186.48 & 0.84 & 199.6170 & 156.6432 & 78.47187 \\
7 & 12.66 & 15.75 & 186.20 & 0.82 & 199.3950 & 152.6840 & 76.57364 \\
8 & 12.64 & 15.70 & 186.50 & 0.84 & 198.4480 & 156.6600 & 78.94259 \\
9 & 12.60 & 15.74 & 186.52 & 0.85 & 198.3240 & 158.5420 & 79.94090 \\
10 & 12.68 & 15.77 & 186.48 & 0.81 & 199.9636 & 151.0488 & 75.53815 \\
11 & 12.70 & 15.74 & 186.70 & 0.82 & 199.8980 & 153.0940 & 76.58606 \\
12 & 12.68 & 15.75 & 186.30 & 0.82 & 199.7100 & 152.7660 & 76.49392 \\
13 & 12.71 & 15.78 & 186.50 & 0.81 & 200.5638 & 151.0650 & 75.32017 \\
14 & 12.68 & 15.76 & 186.60 & 0.84 & 199.8368 & 156.7440 & 78.43600 \\
15 & 12.65 & 15.68 & 186.50 & 0.82 & 198.3520 & 152.9300 & 77.10031 \\
16 & 12.68 & 15.76 & 186.40 & 0.81 & 199.8368 & 150.9840 & 75.55365 \\
\hline \multicolumn{7}{c}{ Average } \\
\hline \multicolumn{7}{c}{}
\end{tabular}




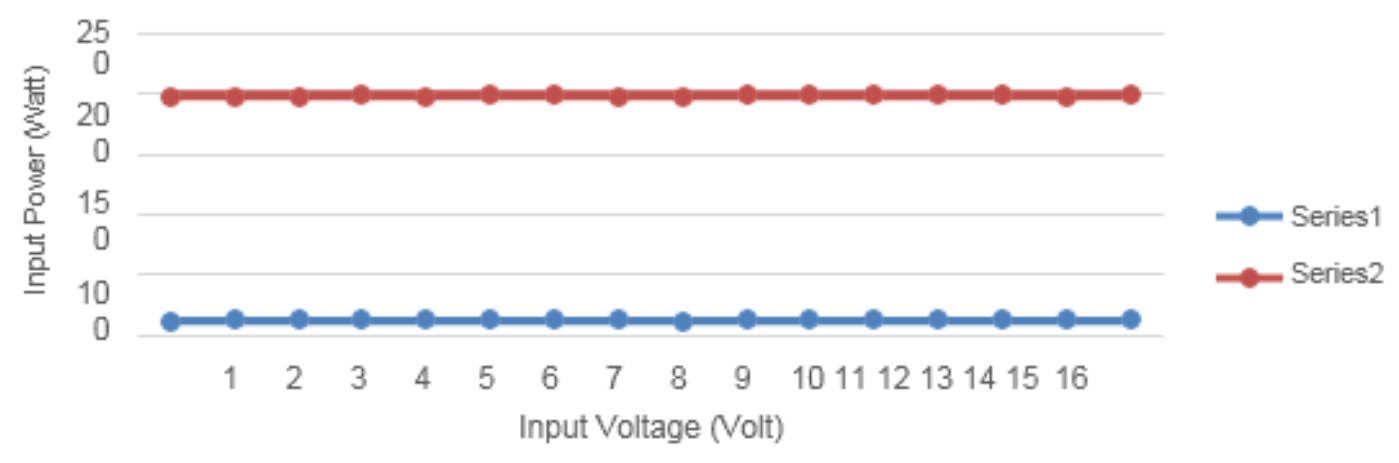

Figure 8. Graph V Input vs Input Power with 200 Watt load

Table 6. Testing Data of Pure Sine Inverter DC to AC with 240 Watt Load

\begin{tabular}{|c|c|c|c|c|c|c|c|}
\hline No & $\mathbf{V}_{\text {in }}(\mathbf{V})$ & $\mathbf{I}_{\text {in }}(\mathbf{A})$ & $\mathbf{V}_{\text {out }}(\mathbf{V})$ & $\mathbf{I}_{\text {out }}(\mathbf{A})$ & $\mathbf{P}_{\text {In }}$ & $\mathbf{P}_{\text {Out }}$ & $\begin{array}{c}\text { Efficiency } \\
(\%)\end{array}$ \\
\hline 1 & 12.56 & 19.90 & 175.2 & 1.07 & 249.944 & 187.464 & 75.00240 \\
\hline 2 & 12.56 & 19.87 & 175.9 & 1.08 & 249.567 & 189.972 & 76.12058 \\
\hline 3 & 12.55 & 19.80 & 175.7 & 1.06 & 248.490 & 186.242 & 74.94949 \\
\hline 4 & 12.56 & 19.87 & 175.7 & 1.08 & 249.567 & 189.734 & 76.02538 \\
\hline 5 & 12.53 & 19.94 & 175.2 & 1.07 & 249.848 & 187.464 & 75.03116 \\
\hline 6 & 12.54 & 19.70 & 175.2 & 1.10 & 247.038 & 192.720 & 78.01229 \\
\hline 7 & 12.58 & 19.86 & 175.9 & 1.09 & 249.839 & 191.731 & 76.74188 \\
\hline 8 & 12.57 & 19.84 & 175.6 & 1.08 & 249.389 & 189.648 & 76.04512 \\
\hline 9 & 12.54 & 19.80 & 175.7 & 1.08 & 248.292 & 189.756 & 76.42453 \\
\hline 10 & 12.55 & 19.90 & 175.1 & 1.08 & 249.745 & 189.108 & 75.72043 \\
\hline 11 & 12.54 & 19.68 & 175.5 & 1.05 & 246.787 & 184.275 & 74.66959 \\
\hline 12 & 12.53 & 19.70 & 175.5 & 1.09 & 246.841 & 191.273 & 77.48842 \\
\hline 13 & 12.56 & 19.88 & 175.0 & 1.05 & 249.693 & 183.750 & 73.59043 \\
\hline 14 & 12.57 & 19.87 & 175.7 & 1.08 & 249.766 & 189.756 & 75.97354 \\
\hline 15 & 12.54 & 19.89 & 175.6 & 1.05 & 249.421 & 184.380 & 73.92332 \\
\hline 16 & 12.56 & 19.70 & 175.8 & 1.09 & 247.432 & 191.622 & 77.44431 \\
\hline \multicolumn{7}{|c|}{ Aver: } & 75.82268 \\
\hline
\end{tabular}

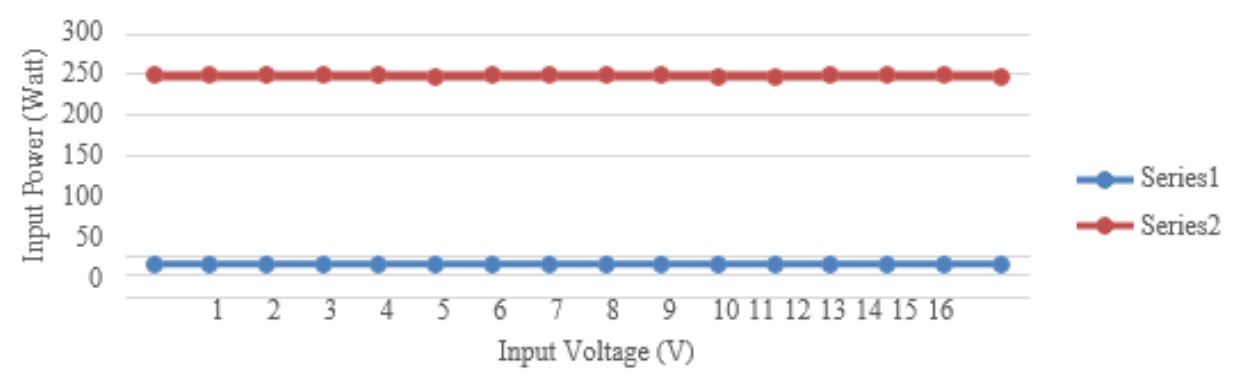

Figure 9. Graph V Input vs Input Power with 240 Watt load 


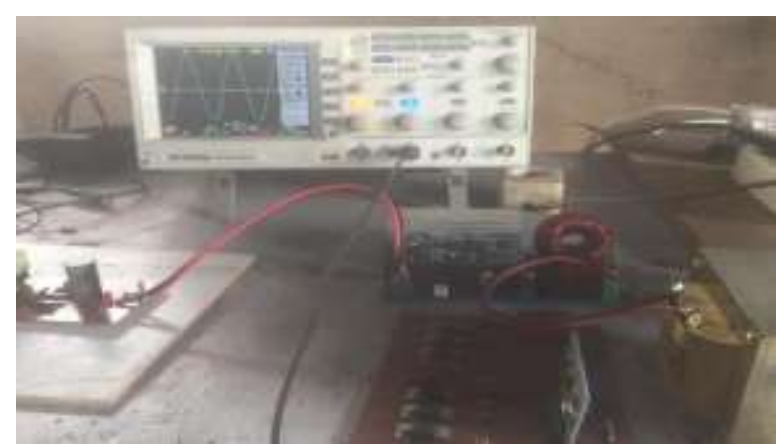

Figure 10. Testing tools

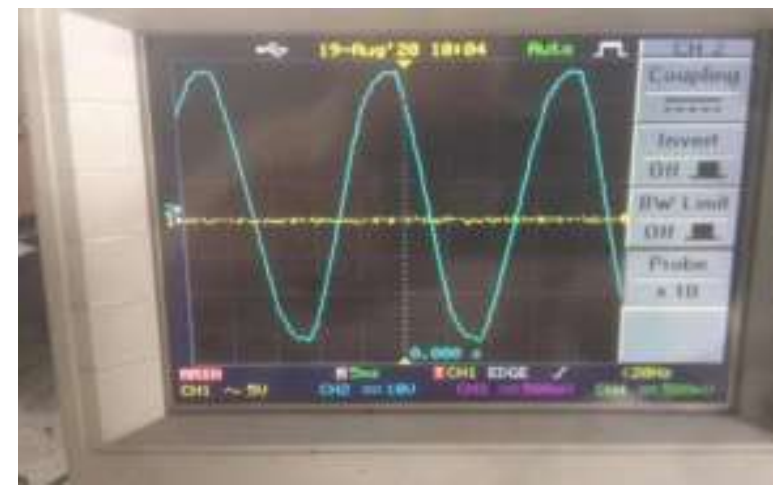

Figure 11. Waveform testing a pure sine wave inverter

\section{Conclusion}

In this research, it can be concluded that it has succeeded in designing a pure sine wave inverter using the ASIC (Application Specific Integrated Circuit) method. The results of testing the load using the EG8010 Microcontroller can produce a Pure Sinus form with different load power conditions of 40, 75, 100, 150, 200, 240 Watt. When the load is 240 Watt, the inverter output voltage drops to 175 Volt. This is because it is linear with increasing load. Then the benefits generated from the pure sinus inverter are that it can be used for household electronic devices, or other electronic devices. With its output in the form of pure sinus, it will be much safer to use to power electronic equipment.

\section{REFERENCES}

[1] B. Stephen Pure Sine Wave Inverter, Woprcester Polytechnic Institute Qualufying Project, 2011.

[2] Francisco. C. D. L. R, Harmonics And Power Systems, CRC press, 2006.

[3] Doucet. J, Eggleston. D, and Shaw. J, DC/AC Pure Sine Wave Inverter, PFC Worcester Polytecnic Institute, 2007.

[4] Merwe. G. J, 150W Inverter-An Optimal Design For Use In Solar Home Systems.

[5] Hart, D. Introduction Power Hall. Electronic Upper Saddle River, NJ: Prentice, 1997.

[6] M. Maina, A Pure Sine Wave Inverter For House Backup, Univertsity Nairobi, 2009. 
[7] T. Sutikno, Pembangkit Sinyal PWM Sinusoidal Dua Fasa Berbasis FPGA, Program Studi Teknik Elektro, Program Pasca Sarjana, Universitas Gajah Mada, Yogyakarta, 2004.

[8] S. Indra, Mikrokontroler Menguasai Arduino, Yogyakarta, Teknosain, 2018.

[9] J. S. S. Michael, Apllicaton Specific Integrated Circuits, San Fransisco: Addison Wesley Longman, 1997.

[10] M. Cahyadi, E. Nasrullah, and A. Trisanto, Rancang Bangun Catu Daya DC 1V - 20V Menggunakan Kendali P-I Berbasis Mikrokontroler, J. Rekayasa dan Teknol Elektro, vol. 10, no. 2, 2016.

[11] National Instruments, Multisim User Guide, Electronics Workbench, 2007.

[12] Sulasno, Teknik Konversi Energi Listrik Dan Sistem Pengaturan, Yogyakarta, Graha, 2013.

[13] Y. Haroen, Elektronika Daya Lanjut, EL 642, Jurusan Teknik Elektro - FTI, Institut Teknologi Bandung, 1998.

[14] Y. Tanoto, Simulasi Filter Pasif Dan Perbandingan Unjuk Kerjanya dengan Filter Aktif Hibrid Dalam Meredam Harmonisasi pada Induction Furnace, Jurnal Teknik Elektro, Vol. 5, UK Petra, Surabaya, 2005. 